Document downloaded from:

http://hdl.handle.net/10251/83421

This paper must be cited as:

Gómez Paricio, A.; Santiago Portillo, A.; Navalón Oltra, S.; Concepción Heydorn, P.; Alvaro Rodríguez, MM.; García Gómez, H. (2016). MIL-101 promotes the efficient aerobic oxidative desulfurization of dibenzothiophenes. Green Chemistry. 18(2):508-515. doi:10.1039/C5GC00862J

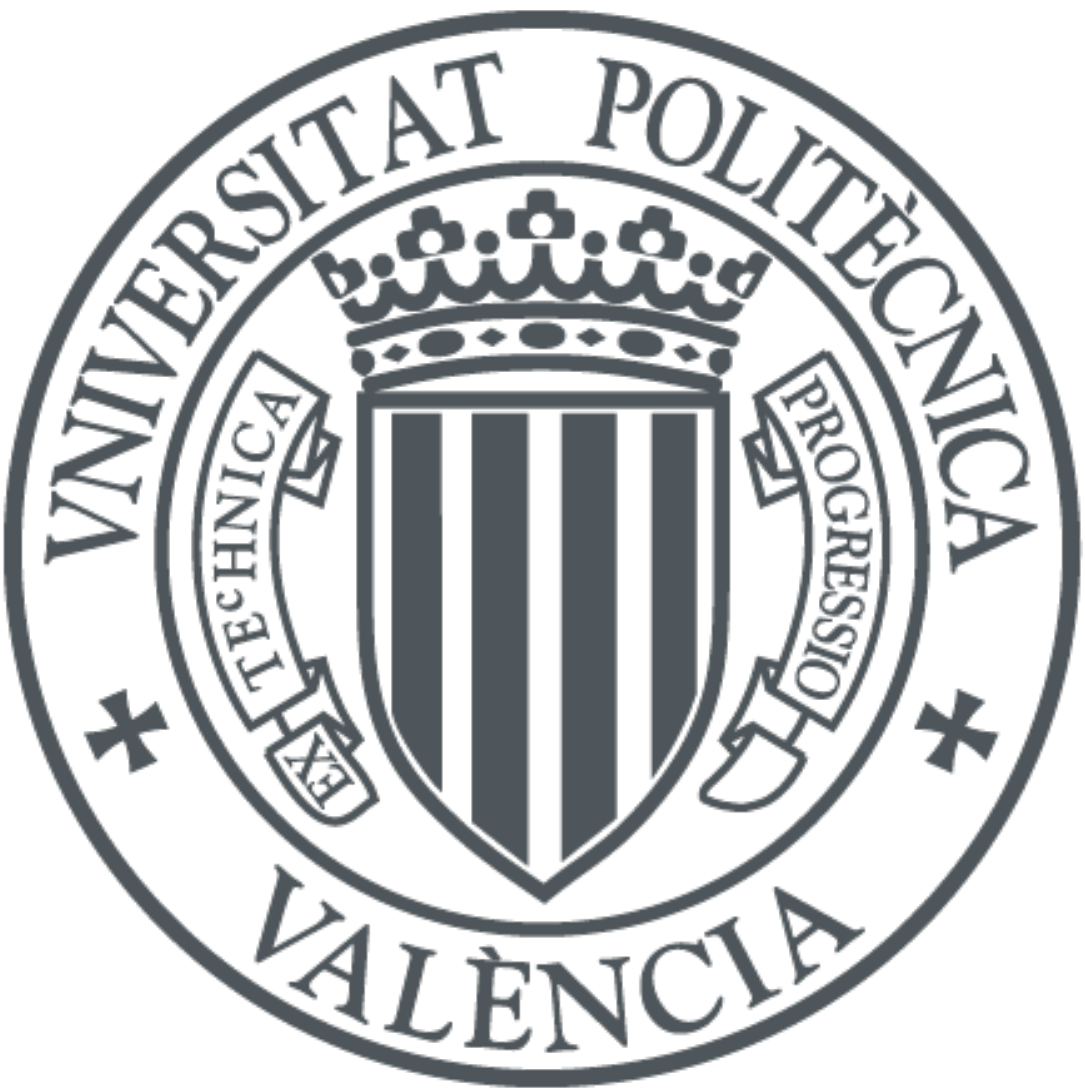

The final publication is available at http://doi.org/10.1039/c5gc00862j

Copyright Royal Society of Chemistry

Additional Information 


\title{
MIL-101 promotes the efficient aerobic oxidative desulfuration of dibenzothiophenes
}

\author{
Adrián Gómez-Paricio, ${ }^{a}$ Andrea Santiago-Portillo, ${ }^{a}$ Sergio Navalón, ${ }^{a}$ Patricia Concepción, ${ }^{\mathrm{b}}$ \\ Mercedes Alvaro ${ }^{a}$ and Hermenegildo Garcia ${ }^{\text {b,c,* }}$
}

\begin{abstract}
MIL-101 promotes the aerobic oxidation in $n$-dodecane of dibenzothiophene (DBT) and its methyl-substituted derivatives to their corresponding sulfone with complete selectivity, without observation of the sulfoxide. DBT sulfones can be completely separated from $n$-dodecane by water extraction. MIL-101(Cr) without the need of pre-activation was found more convenient than the also-active MIL-101(Fe) analog. The reaction exhibits an induction period due to the diffusion inside the pore system of the solvent or oxygen and it is not observed if the MIL-101 sample is first contacted with the solvent at the reaction temperature for sufficiently long times. MIL-101 is reusable for at least five times without any sign of deactivation according to the time-conversion plots. Evidence by electron paramagnetic resonance spectroscopy detecting the hydroperoxide radical adduct with a spin trapping agent and Raman spectroscopy detection of superoxide supports that the process is an autooxidation reaction initiated by MIL-101 following the expected radical chain mechanism inside the MIL-101 cages.
\end{abstract}

\section{Introduction}

Metal organic frameworks (MOFs) are finding increasing application as heterogeneous catalysts, particularly for liquid phase reactions in which some MOFs exhibit a remarkable stability allowing their recovery and reuse in many consecutive runs. ${ }^{1-7}$ MOFs are constituted by nodes of metal ions or small clusters of metal ions held in place by rigid bi- or multipodal organic linkers and offer in catalysis a large tunable porosity, a high density of active sites and a large variety on the transition metals that can be employed. ${ }^{1,8}$ " Among the various MOFs that have been reported MIL-101 has been extensively used because it has shown a considerable robustness under catalytic conditions, it can be prepared reliably in sufficient quantities and it has a BET surface area above $2,000 \mathrm{~m}^{2} \mathrm{~g}^{-1}$ and a high metal content ( 20 wt \%). ${ }^{6,10-12}$ There are in the literature some examples of the use of MOFs as catalysts to promote oxidation reactions although most of them use organic peroxides such as tert-butylhydroperoxide (TBHP) $)^{13-19}$ or $\mathrm{H}_{2} \mathrm{O}_{2}{ }^{19}, 20$ as terminal oxidants or as initiators when combined with molecular oxygen. ${ }^{16,17,21,22}$ Interestingly, in a series of previous studies we have shown that MOFs are suited

\footnotetext{
a. Departamento de Química, Universidad Politécnica de Valencia, C/Camino de Vera, $s / n, 46022$ Valencia, Spain.

${ }^{b}$. Instituto de Tecnología Química CSIC-UPV, Universidad Politécnica de Valencia, Av. de los Naranjos s/n, 46022 Valencia, Spain.

c. Center of Excellence for Advanced Materials Research, King Abdulaziz University, Jeddah, Saudi Arabia

† Footnotes relating to the title and/or authors should appear here.

Electronic Supplementary Information (ESI) available: [details of any supplementary information available should be included here]. See DOI: $10.1039 / \times 0 \times x 00000 x$
}

catalysts for the aerobic oxidations of benzylic compounds ${ }^{23}$ and thiols ${ }^{24}$ or when combined with radical initiators such as N-hydroxyphthalimide $(\mathrm{NHPI})^{25-27}$ or 2,2,6,6-tetramethyl-1piperidinyloxy (TEMPO) $)^{28}$ able to promote the aerobic oxidation of different substrates including saturated and unsaturated hydrocarbons, ${ }^{25,}{ }^{27}$ alcohols $^{28}$ and amines. ${ }^{26}$ The use of molecular oxygen as terminal oxidant replacing other reagents is very convenient from the point of view of the process greenness and for the economic competitiveness of the reaction. ${ }^{6,29}$ In this context, in the present manuscript we report that dibenzothiophenes (DBTs) can be efficiently oxidized by molecular oxygen in the presence of MIL-101. DBT and its methyl-substituted derivatives are among the sulfur compounds present in gasoil and high boiling-point oil distillates and they are responsible for a large percentage of SOx atmospheric pollution. ${ }^{30}$ The tendency of legal regulations on fuel quality is to decrease the sulfur content $(<10$ ppmw $S$, part per million weight of sulfur) and conventional hydrodesulfuration treatments in refining have to be supplemented with additional treatments to remove in higher degree sulfur containing compounds present in fuels. ${ }^{30,}{ }^{31}$ It should be noted that DBTs, in particular 4-methyl DBT and 4,6dimethyl DBT, are among the most reluctant sulfur compounds to undergo hydrodesulfuration. ${ }^{18,30,31}$ One possibility to remove DBT and its alkyl derivatives from oil distillates is their oxidation to the corresponding sulfoxides and/or sulfones that have an increased hydrophilicity and higher boiling point, thus, making easier their removal from fuels. ${ }^{30}$ Typically, in these oxidative treatments organic hydroperoxides are used as oxidation reagents in combination with transition metal containing catalysts. ${ }^{18,} 30,32-37$ It would be advantageous considering the large volumes of fuels to be treated if 
hydroperoxides are replaced by molecular oxygen. Some attemps to use molecular oxygen have been reported using polyoxometalates. For example, a composite of nanocrystalline MOF (NENU-9N) impregnated with a phosphovanadomolybdate has been employed to promote a ultradeep oxidative desulfuration at $80{ }^{\circ} \mathrm{C}$ for $1.5 \mathrm{~h}$. The role of the MOF is to facilitate the dispersion of the hydrophilic polyoxometalate in the model or real diesel environment. It is important to note, however, that the catalytic oxidation was carried out in the presence of a large excess of isobutyraldehyde respect to DBT (10:1 molar ratio). It was proposed that isobutrylaldehyde becomes oxidized to the corresponding peracid by molecular $\mathrm{O}_{2}$ and then, in a second step, the peracid promotes the oxidation of DBT to DBTsulfone. Under the studied conditions a TOF of $80 \mathrm{~h}^{-1}$ was obtanied. In other work, an Anderson-type polyoxometalate modified with quaternary ammonium salts was employed for the aerobic oxidation of DBT-derivatives using molecular $\mathrm{O}_{2}$ as oxidant. The role of the quarternary ammonium salt is to facilitate the dispersión of the polyoxometalate in decalin as reaction medium, otherwise, the catalytic activity of the polyoxometalate is neglibible. Good catalytic activity of the system were achieved, while the turnover frequency achieved only achieves $12.8 \mathrm{~h}^{-1}$. However, details of how the quaternary ammonium salt is recovered from the reaction mixture and reusability tests and catalyst stability were not reported and, therefore, the applicability of the materials still needs further research.

Besides hydrodesulfuration or oxidative desulfuration removal of DBT derivatives from oils can be carried out by adsorption methods. ${ }^{38}$ Carbonaceous materials (activated carbon, carbon aerogels or carbon nanotubes), ${ }^{39,40}$ metal or metal oxides, ${ }^{40}$ zeolites, ${ }^{40}$ clays, ${ }^{41-43}$ silicas, ${ }^{44}$ organic

polymers $^{40}$ or metal coordination polymers ${ }^{31,45}$ have been used among others as adsorbents. In addition, molecular imprinting technology has also been applied for the selective desulfurization of oils. ${ }^{3}$

In the present paper we report that $\mathrm{MIL}-101(\mathrm{Cr})$ $\left(\mathrm{Cr}_{3} \mathrm{~F}\left(\mathrm{H}_{2} \mathrm{O}\right)_{2} \mathrm{O}\left[\left(\mathrm{O}_{2} \mathrm{C}\right)-\mathrm{C}_{6} \mathrm{H}_{4}-\left(\mathrm{CO}_{2}\right)\right]_{3} \cdot \mathrm{nH}_{2} \mathrm{O}\right) \quad(\mathrm{n} \leq 25)$ promotes the transformation of DBT and methyl-substituted derivatives into the corresponding sulfones by just by molecular oxygen without the need of aldehyde or ammonium salts. MIL$1001(\mathrm{Cr})$ can be recovered and reused without decrease of the catalytic activity. The present finding may be advantageous respect to those oxidative desulfurations requiring hydroperoxides as oxidants or the addition of excess amounts of additives.

\section{Results and discussion}

For the present study two MIL-101 materials, namely MIL101(Cr) and MIL-101(Fe), were used. These two materials are isostructural differing in the nature of the transition metal and the presence of $-\mathrm{F}$ or $-\mathrm{Cl}$ as ligand in the case of MIL-101(Cr) and MIL-101(Fe), respectively. ${ }^{9,}$ BET surface areas and pore volumes obtained after synthesis were around $1,850 \mathrm{~m}^{2} \mathrm{~g}^{-1}$ and $1.12 \mathrm{~cm}^{3}$, respectively. ${ }^{23}$ These differences can influence the interaction of the MOF with molecular oxygen and can also modify the polarity of the internal voids of the material. ${ }^{12,23,47}$ It has been shown that differences in polarity of the starting compound and the resulting oxidized products determine

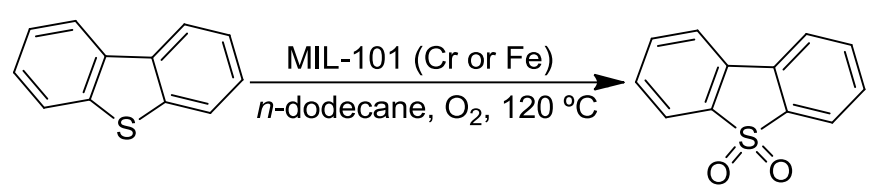

significant variations in the affinity for the internal surface, this resulting in changes in product selectivity. ${ }^{23}$ In addition, MIL101 solids exhibit large pores (free diameters of 29 and $34 \AA$ ) with microporous windows of 12 and $16 \AA$, respectively, allowing the adsorption of DBT derivatives into their cavities. ${ }^{48}$ In the initial stage of our work we performed treatment of DBT $\left(200 \mathrm{mg} \mathrm{L}^{-1}\right.$ ) dissolved in $n$-dodecane at $120{ }^{\circ} \mathrm{C}$ (flash point and auto-ignition temperatures $\sim 71{ }^{\circ} \mathrm{C}$ and $\sim 203{ }^{\circ} \mathrm{C}$, respectively) with $\mathrm{O}_{2}$ in the presence of MIL-101(Cr or Fe) without or with pre-activation treatment (Scheme 1). The conditions used are outside the explosion range.

Scheme 1 Aerobic oxidation of DBT to DBT-sulfone using MIL$101(\mathrm{Cr}$ or $\mathrm{Fe})$ as catalyst.

It has been frequently observed that, in order to be used as catalyst, MOFs have to be submitted to an activation process to remove co-adsorbed water and solvent molecules and to generate coordinatively unsaturated sites around the metal centers. ${ }^{13,22,49,50}$ In the present case, MIL-101 solids were activated at $150{ }^{\circ} \mathrm{C}$ under vacuum for $6 \mathrm{~h}$ and the performance of the materials submitted to activation compared with that of untreated materials. In all cases it was observed that the only reaction product was the corresponding DBT sulfone (DBTsulfone) and the process is accompanied by some oxidation of the solvent ( $1 \%)$. In the absence of catalyst, autooxidation occurs in a negligible extent, even if the temperature is increased up to $180{ }^{\circ} \mathrm{C}$ (Figure S1). It was observed that this activation treatment does not influence the performance of MIL-101(Cr), while the activity for MIL-101(Fe) increases upon activation. It has been reported that under these activation conditions $\mathrm{Fe}^{3+}$ becomes reduced to $\mathrm{Fe}^{2+}$ in $\mathrm{MIL}-101(\mathrm{Fe}){ }^{51-53}$ while this reduction process has not been observed for the case of MIL-101(Cr). ${ }^{12}$ Also, coordinated water is easier desorbed from MIL-101(Fe) than in MIL-101(Cr). ${ }^{12,}{ }^{47}$ Comparison between the two MOFs shows that MIL-101(Fe) is less active than MIL-101(Cr), although the performance of the two samples is similar (Figure 1). In one of these experiments, $n$-dodecane containing DBT (200 $\mathrm{mg} \mathrm{L}^{-1}$ as S, 267 ppmw S) was submitted to oxidation by MIL-101(Cr) at $120{ }^{\circ} \mathrm{C}$ and after the reaction the catalyst was filtered and the resulting clear $n$ dodecane solution extracted with water leading to the complete removal from $n$-dodecane of sulfur below to the detection limit ( $4 \mathrm{mg} \mathrm{L}^{-1}, \sim 5.3 \mathrm{ppmw}$ ), indicating that upon transformation of DBT into DBT-sulfone the latter compound can be completely extracted by water. 


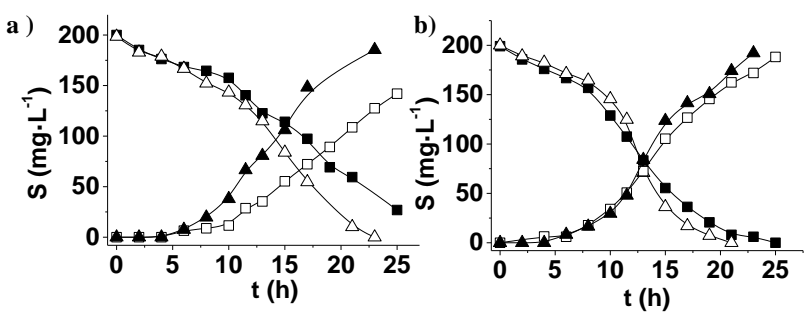

Figure 1 Aerobic oxidation of DBT to DBT-Sulfone using (a) MIL-101(Fe) or (b) MIL-101(Cr) with or without activation. Reaction conditions: Catalyst $\left(500 \mathrm{mg} \mathrm{L}^{-1}\right)$, DBT $\left(1,150 \mathrm{mg} \mathrm{L}^{-1}\right.$, $1,534 \mathrm{ppmw}), n$-dodecane $(10 \mathrm{~mL}), \mathrm{O}_{2}(1 \mathrm{~atm}), 120^{\circ} \mathrm{C}$. Legend using non-activated catalyst, DBT ( $\square$ ) and DBT-sulfone ( $\square)$, or using activated catalyst DBT $(\Delta)$, DBT-sulfone $(\boldsymbol{\Delta})$.

A notable observation of the temporal evolution of the reaction was the presence of an induction period (Figure 1). To understand the origin of this induction period, several tests were performed including (Figure 2): 1) heating a solution of DBT in $n$-dodecane in the presence of MIL-101(Cr) under Ar for $20 \mathrm{~h}$ and then admitting $\mathrm{O}_{2}, 2$ ) heating MIL-101(Cr) in $n$ dodecane under $\mathrm{O}_{2}$ atmosphere for $12 \mathrm{~h}$ and then adding DBT, and 3 ) heating $\mathrm{MIL}-101(\mathrm{Cr})$ in $n$-dodecane under $\mathrm{Ar}$ atmosphere for $12 \mathrm{~h}$ and then sudden addition of DBT under $\mathrm{O}_{2}$ atmosphere. In all cases the initial induction period taking about $6 \mathrm{~h}$ was not longer observed. This indicates that the diffusion of DBT or oxygen activation are not responsible for the induction period, but most probably the diffusion of $n$ dodecane inside the MIL-101 pores. Overall the above control reactions indicating that the induction period can be avoided by melting MIL-101 and $n$-dodecane at the required temperatura before starting the oxidation. In a related adsorption study of linear alkanes, a change in the adsorption regime along the chain length was observed for $n$-nonane, whose adsorption takes a significantly longer equilibration time. ${ }^{54}$ This slow down in the adsorption time from $n$-nonane was attributed as reflecting the large conformational freedom for this linear alkane and their relative dimensions with respect to the size of MIL-101 pores. ${ }^{54}$ Similarly here, diffusion of $n$ dodecane inside MIL-101 will be a slow process influencing the reaction kinetics.
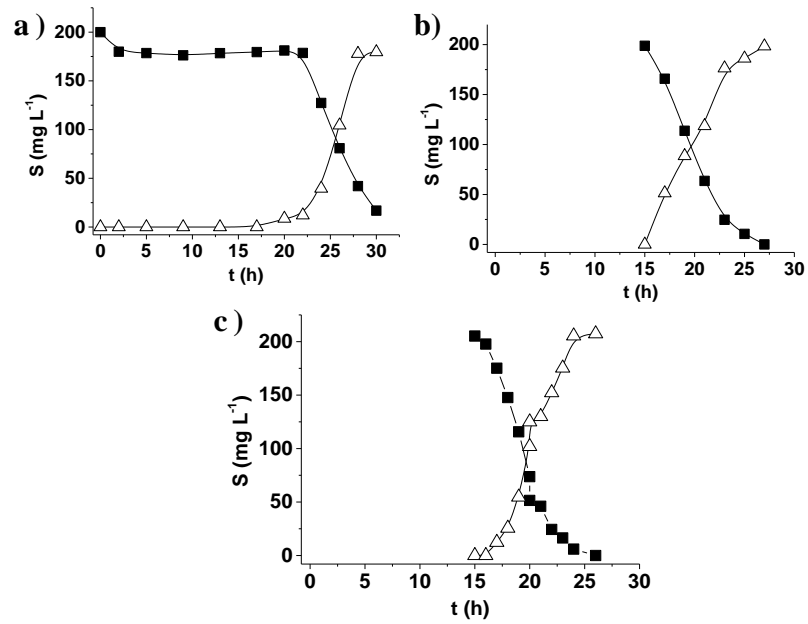

Figure 2 Influence on the aerobic oxidation of DBT ( $\square$ ) to DBTsulfone $(\Delta)$ using $\mathrm{MIL}-101(\mathrm{Cr})$ as catalyst at various pretreatments. Reaction conditions: Catalyst (500 $\mathrm{mg} \mathrm{L}^{-1}$ ), DBT $\left(1,150 \mathrm{mg} \mathrm{L}{ }^{-1}\right), n$-dodecane $(10 \mathrm{~mL}), 120{ }^{\circ} \mathrm{C}$, atmosphere contacting as a function of the experiment ( $\mathrm{a}, \mathrm{b}$ or $\mathrm{c}$ ). Legend: (a) DBT in $n$-dodecane with MIL-101(Cr) under Ar at $120{ }^{\circ} \mathrm{C}$ for $15 \mathrm{~h}$ and then sudden admission $\mathrm{O}_{2}$; (b) suspending MIL$101(\mathrm{Cr})$ in $n$-dodecane under $\mathrm{O}_{2}$ at $120{ }^{\circ} \mathrm{C}$ for $15 \mathrm{~h}$ and then addition of DBT; (c) contacting MIL-101(Cr) in $n$-dodecane under $\mathrm{Ar}$ for $15 \mathrm{~h}$ at $120{ }^{\circ} \mathrm{C}$ and then sudden addition of DBT under $\mathrm{O}_{2}$ atmosphere.

Considering the above explanation for the induction period in the aerobic oxidation of DBT and the convenience of prior suspension in of MIL-101(Cr) in the solvent, we envisioned the possibility that the chain length of the solvent could play a role on the reaction kinetics. As it can be seen in Figure S2 the temporal profile of the DBT oxidation clearly depends on the solvent, following the expected trend that shorter $n$-alkane length decreases the induction period.

The influence of oxygen pressure and reaction temperature on the aerobic DBT oxidation promoted by MIL-101(Cr) was also studied (Figures S3 and S4). It was found that oxygen concentration influences the induction period, but not the reaction rate after initiation. Thus, the reaction can be suitably carried out under air. This can be interpreted considering that besides $n$-dodecane diffusion, oxygen diffusion also contributes to the induction period that is resulting from the faster of these two process. When oxygen pressure increases, oxygen can access faster to the metal centers and form the superoxide that will react with DBT outside MIL-101 crystallites. Whatever is faster, either filling of the microporoes by $n$-dodecane or diffusing of the reactive oxygen species outside the MIL-101 crystalline will initiate DBT oxidation. As expected the induction period decreases and the reaction rate increases along with the reaction temperature in the range from 110 to $140{ }^{\circ} \mathrm{C}$. The Arrhenius plot of the natural logarithm of the rate after the induction period follows a linear relationship with the inverse of the absolute temperature, that allows estimation of an apparent activation energy (Ea) of 137 $\mathrm{kJ} \mathrm{mol}^{-1}$. This Ea value is higher than those reported in the literature for analogous DBT oxidation by TBHP using MIL$47(\mathrm{~V})$ or MIL-125(Ti) that were estimated as 51 and $75 \mathrm{~kJ} \mathrm{~mol}^{-1}$, respectively. ${ }^{32}$ Considering that organic peroxides are more reactive than molecular oxygen, the higher Ea value is not unexpected for the present aerobic oxidation. One additional experiment in where the amount of catalyst is increased resulted in a further decrease of the induction period as well as the final reaction time. Complete DBT conversions could be achieved in $4 \mathrm{~h}$ reaction time (compare Figure S4d and Figure S5).

MIL-101(Cr) was found to be stable as catalyst with respect to its activity. Figure 3 shows the temporal profile of DBT disappearance and DBT-sulfone formation in five consecutive runs using the same MIL-101(Cr) sample that was recovered from the reaction mixture by filtration washed with water and recycled. As it can be seen in Figure 3, exactly the same 
temporal profile was reproduced in these five consecutive runs, indicating that no catalyst deactivation is taking place. This result was also confirmed by ICP-OES analysis of the liquid phase after the reaction that shows that the chromium content was below $0.5 \%$ of the total initial $\mathrm{Cr}$ present in the fresh catalyst. Blank control experiments using similar amounts of leached $\mathrm{Cr}^{3+}$ in the absence of MIL-101(Cr) reveals that the reaction is promoted by $\mathrm{MIL}-101(\mathrm{Cr})$ with negligible activity measured for the small amount of leached chromium (Figure S6). In addition, XRD and ATR-FT-IR of the MIL-101(Cr) fresh, three and five times used were coincident indicating that the crystal structure remaines unaltered during the process (Figure S7). These findings on MIL-101(Cr) stability agrees with previous results that also employed this material as catalyst and noticed its remarkable stability. ${ }^{16,22,23}$
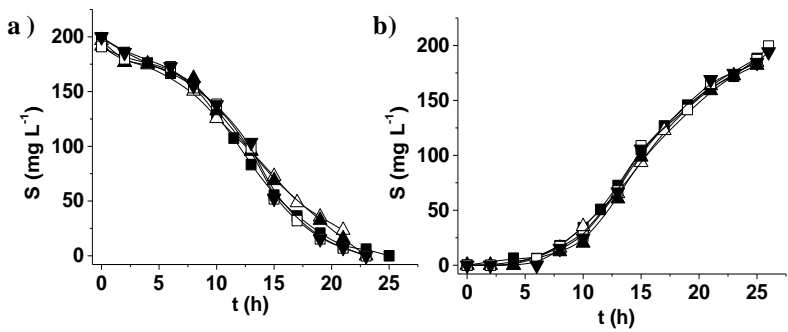

Figure 3 Reusability of MIL-101(Cr) as catalyst in the aerobic oxidation of DBT (a) to DBT-sulfone (b). Reaction conditions: catalyst (500 $\left.\mathrm{mg} \mathrm{L}^{-1}\right)$, DBT $\left(1,150 \mathrm{mg} \mathrm{L}^{-1}, \sim 1,534 \mathrm{ppmw}\right), n$ dodecane $(10 \mathrm{~mL}), \mathrm{O}_{2}(1 \mathrm{~atm}), 120^{\circ} \mathrm{C}$. Legend: $1^{\text {st }}$ use $(\mathbf{\square}), 2^{\text {nd }}$ use $(\Delta), 3^{\text {th }}$ use $(\boldsymbol{\Delta}), 4^{\text {th }}$ use $(\square), 5^{\text {th }}$ use $(\boldsymbol{\nabla})$.

The catalyst stability was further supported by performing an additional catalytic test in which five times higher DBT amount was submitted to oxidation using 50 times lower catalyst amount and observing and almost complete disappearance of DBT at sufficiently long periods of time (Figure S8). It was noticed, however, in this experiment that a gradual precipitation of DBT-sulfone as solid develops as the reaction progresses as consequence of the low solubility of DBT-sulfone in $n$-dodecane.

Heterogeneity of the process was studied by carrying out two twin experiments and removing the catalyst in one of them once the induction period was overcome (10 h) (Figure 4). Exactly the same temporal profiles for DBT disappearance and DBT-sulfone formation were observed in these two experiments indicating that once the reaction has started the presence of MIL-101(Cr) is not longer necessary. This type of behavior is common for radical chain autooxidation reactions in where the role of the initiator is to generate the first radicals that subsequently undergo a propagation cycle by reaction with molecular oxygen that does not requires any catalyst. ${ }^{23,55}$ ${ }^{56}$ To provide some support to this autooxidation mechanism, TEMPO was added as radical trap, observing that in the presence of this radical inhibitor, no DBT oxidation takes place for periods longer than $30 \mathrm{~h}$. On the other hand, if the reaction is started by MIL-101(Cr) in the absence of TEMPO and then, once the reaction progresses, TEMPO is added, a significant decrease in the reaction rate is observed (Figure 4).

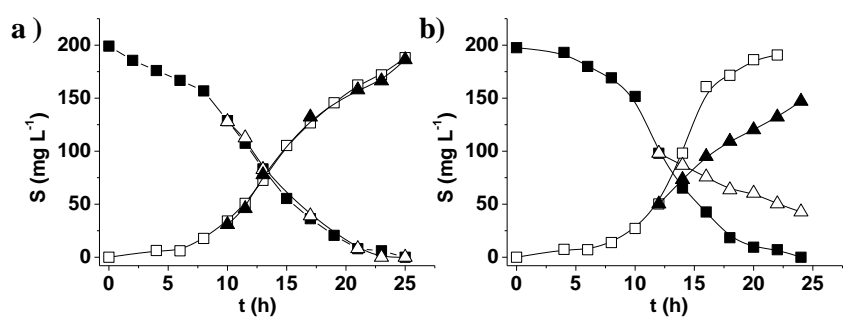

Figure 4 (a) Hot filtration test at $38 \%$ conversion for the aerobic oxidation of DBT to DBT-sulfone using MIL-101(Cr) as catalyst. Reaction conditions: Catalyst $\left(500 \mathrm{mg} \mathrm{L}^{-1}\right)$, DBT $(1,150$ $\left.\mathrm{mg} \mathrm{L}^{-1}\right), n$-dodecane $(10 \mathrm{~mL}), \mathrm{O}_{2}(1 \mathrm{~atm}), 120^{\circ} \mathrm{C}$. Legend: In the presence of catalyst DBT ( $\square)$, DBT-sulfone ( $\square$ ); after catalyst filtration of the catalyst DBT $(\Delta)$, DBT-sulfone $(\boldsymbol{\Delta})$. b) Hot filtration test at $38 \%$ conversion in the presence of TEMPO (20 mol \% respect DBT). Legend: in the presence of catalyst DBT $(\square)$, DBT-sulfone $(\square)$; after catalyst filtration in the presence of $\operatorname{TEMPO}$ DBT $(\Delta)$, DBT-sulfone $(\boldsymbol{\Delta})$.

According to these data, the following autooxidation mechanism shown in Scheme 2 is proposed. In this mechanism after initiation by generation of the first $\mathrm{DBT}^{++}$, a propagation chain occurs by reaction with molecular oxygen, formation of DBT- $\mathrm{O}_{2}^{++}$and electron abstraction from neutral DBT. The first $\mathrm{DBT}^{++}$radical would be generated by some reactive oxygen species formed by interaction of the $\mathrm{Cr}^{3+}$ with $\mathrm{O}_{2}$, the most probable one being superoxide $\left(\mathrm{O}_{2}{ }^{-}\right) \cdot{ }^{34,57,58}$

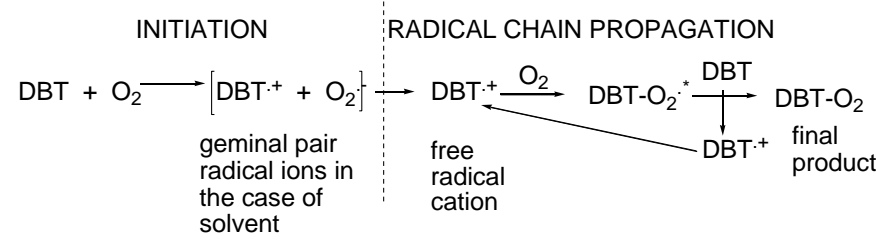

Scheme 2 Proposed reaction mechanism for the aerobic oxidation of DBT with molecular $\mathrm{O}_{2}$ in the presence of MIL$101(\mathrm{Cr})$ as catalyst.

To provide experimental evidence to the generation of reactive oxygen species, EPR spectra using phenyl $\alpha$-tert-butyl nitrone (PBN) as radical trap were carried out. As Figure 5 shows while MIL-101(Cr) and $\mathrm{O}_{2}$ exhibits only a weak EPR signal when PBN is added the EPR signal attributed to the adduct of $\mathrm{PBN}-\mathrm{OOH}$ was recorded. This $\mathrm{HOO}^{\circ}$ radical will be formed from $\mathrm{O}_{2}{ }^{--}$by protonation by moisture present in the medium and constitutes and experimental evidence of the involvement of the generated reactive oxygen species by acting MIL-101(Cr) solid as initiator. ${ }^{23}$ Selective radical quenching experiments using $p$-benzoquinone ${ }^{59}$ or DMSO ${ }^{60,61}$ as $\mathrm{O}_{2}{ }^{-} / \mathrm{HOO}$ or $\mathrm{HO}^{\circ}$ quenchers, respectively, support the generation of $\mathrm{O}_{2}{ }^{-\prime} / \mathrm{HOO}$ species from molecular $\mathrm{O}_{2}$. The presence of $p$-benzoquinone completely inhibits the reaction (Figure S9), supporting the generation of $\mathrm{O}_{2}{ }^{-} / \mathrm{HOO}$ radicals, 
while the presence of DMSO (a HO radical quencher) does not affect the reaction profile of DBT oxidation to form DBTsulfone. The oxidation of DBT to DBT-sulfone by $\mathrm{O}_{2}{ }^{-\top} / \mathrm{HOO}$ was further supported by performing additional experiments employing TBHP as oxidant and observing the conversion of DBT to its corresponding DBT-sulfone (Figure S10). In contrast, the use of benzoyl peroxide as oxidant does not promote the oxidation of DBT to DBT-sulfone (Figure S10).

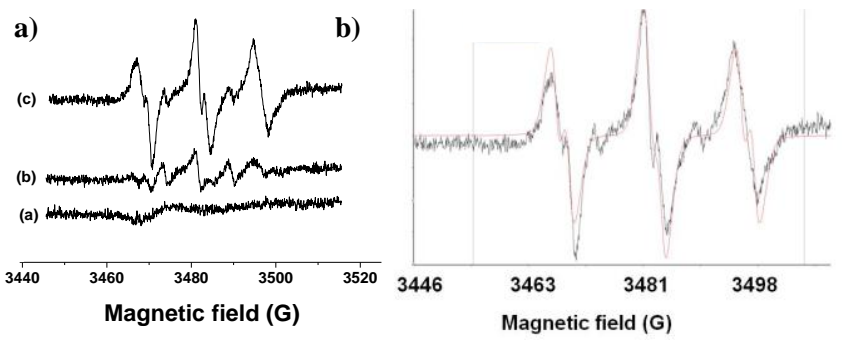

Figure 5 (A) Experimental EPR spectra in $n$-dodecane as solvent at $120{ }^{\circ} \mathrm{C}$ for $5 \mathrm{~h}$ and using (a) MIL-101(Cr) $+\mathrm{O}_{2}$, (b) $\mathrm{PBN}+\mathrm{O}_{2} \mathrm{y}$ (c) MIL-101(Cr) $+\mathrm{PBN}+\mathrm{O}_{2}$. (B) Experimental and simulated EPR spectra of $\mathrm{PBN}-\mathrm{OOH}$ under (c) conditions. Hyperfine coupling constants of $\mathrm{PBN}-\mathrm{OOH}\left(\sim 90 \%\right.$ area) $\mathrm{AG}_{\mathrm{N}}=14.0$ and $A G_{H}=2.05$ and tert-butyl aminoxyl from degraded $P B N A G_{N}=$ 14.0 and $A G_{H}=14.0$.

Besides EPR spectroscopy, Raman also provided valuable information in support of the intermediacy of $\mathrm{O}_{2}{ }^{--}$as the initial reactive oxygen species promoting DBT autooxidation.

Oxygen activation on both MIL-101(Cr) and MIL-101(Fe) samples has been studied by Raman spectroscopy. ${ }^{62-64}$ In the MIL-101(Cr)sample (Figure 6A), two Raman bands at $998 \mathrm{~cm}^{-1}$ and $1035 \mathrm{~cm}^{-1}$ have been detected when the sample was exposed to $\mathrm{O}_{2}$ flow at $120{ }^{\circ} \mathrm{C}$. These bands have been attributed to peroxo and superoxo species, respectively. ${ }^{63}$ Notice that the stability of these bands is relatively weak, since they are easily removed at room temperature by purging the sample with $\mathrm{N}_{2}$. Similar to the MIL-101(Cr) sample, oxygen activation leading to peroxo $\left(826 \mathrm{~cm}^{-1}\right)$, superoxo $\left(1161 \mathrm{~cm}^{-1}\right)$ and $\mathrm{O}_{2}{ }^{-}\left(1313 \mathrm{~cm}^{-1}\right)$ species have been observed in the MIL$101(\mathrm{Fe})$ sample (Figure $6 \mathrm{~B}$ ) when exposed to $\mathrm{O}_{2}$ flow at 120 ${ }^{\circ} \mathrm{C} .{ }^{63,64}$ These species are easily removed by purging with $\mathrm{N}_{2}$ at room temperature. The Raman band at $1502 \mathrm{~cm}^{-1}$ has been ascribed to adsorbed molecular oxygen species, ${ }^{62,63}$ which decrease in intensity by purging with $\mathrm{N}_{2}$. However a different behavior is observed with the $1430 \mathrm{~cm}^{-1}$ Raman band which becomes better resolved after $120{ }^{\circ} \mathrm{C}$ oxygen treatment. Probably this band could be assigned to some structural modification in the MOF framework, indeed, structural modification in the $1650-1400 \mathrm{~cm}^{-1}$ range has already been detected by IR spectroscopy (spectra not shown).

According to these results, oxygen peroxo and superoxo species are generated by molecular oxygen and become stabilized on both MIL-101(Cr) and MIL-101(Fe) samples. The peaks corresponding to these reactive oxygen species in
Raman spectroscopy disappear or decrease significantly if a $\mathrm{N}_{2}$ flow is flushed, indicating that $\mathrm{O}_{2}{ }^{--}$can diffuse away from MIL101 once formed. All these observations are in good agreement with EPR spectroscopy detection of $\mathrm{HOO}^{\circ}$ adduct and the mechanistic proposal shown in Scheme 2.
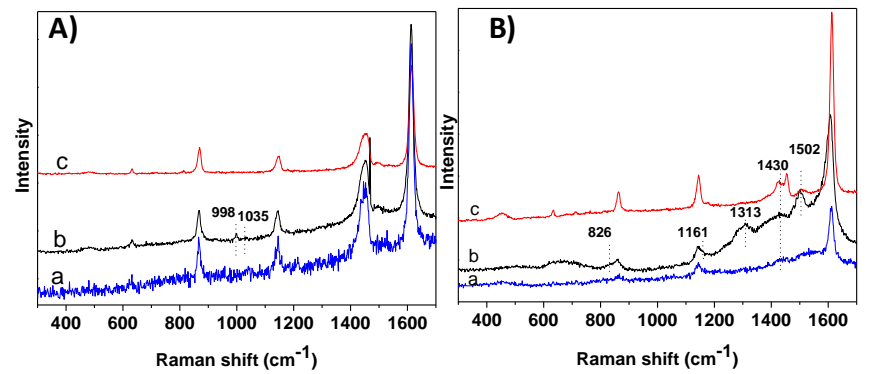

Figure 6 Raman spectra of MIL-101(Cr) (A) and MIL-101(Fe) (B) samples in $\mathrm{N}_{2}$ flow at $120{ }^{\circ} \mathrm{C}(\mathrm{a}), \mathrm{O}_{2}$ flow at $120^{\circ} \mathrm{C}(\mathrm{b})$ and $\mathrm{N}_{2}$ flow at $25{ }^{\circ} \mathrm{C}$ (c).

Furthermore, we investigate the possibility that the sulfur atom of DBT coordinates somehow with the $\mathrm{Cr}^{3+}$ ions of MIL101(Cr), therefore, intervening in the oxidation mechanism. For this purpose, the absorption of DBT in the MIL-101(Cr) was carried out at room temperature and, then, the diffuse reflectance UV-Vis absorption or ATR-FT-IR spectrum of the resulting sample recorded. Figure S11 does not show any change that could be attributable to the interaction between $\mathrm{Cr}^{3+}$ ions of MIL-101(Cr) with DBT. Therefore, the observed adsorption of DBT in MIL-101(Cr) should occur probably by simply $\pi-\pi$ interaction of DBT with MIL-101 organic linkers.

The scope of MIL-101(Cr) as promoter of the aerobic oxidation of aromatic sulfur compounds with DBT structure was checked by carrying out under analogous conditions the oxidation of 4methyl and 4,6-dimethyl substituted DBT (Figure S12). It was observed that these methyl substituted DBT undergo oxidation to the corresponding sulfones exhibiting the same features as parent DBT, but reacting even faster (Figure S12). This reactivity pattern follows the expected order according to the expected influence of electron donor substituents on the electronic density of the $S$ atoms as reported in the literature. ${ }^{65}$ A similar behaviour respect to the oxidation of individual components was observed when working in a multicomponent model solution (Figure S13).

Importantly, the aerobic oxidation of DBT, 4-methyl and 4,6dimethyl DBT to their corresponding sulfones was also efficiently achieved using commercial diesel (Repsol) as medium although we notice that in this case the reaction time is higher respect to the use of $n$-dodecane (Figure S14). This may be explained considering the large number additives that for different purposes are present in commercial diesel and the negative influence that these additives can play for DBT adsorption and reaction.

Finally, the aerobic oxidative process of DBT and its derivatives using MIL-101(Cr) as solid promoter was coupled with a liquidliquid extraction process for the removal of the sulfur 
compounds. The process consists in the simply removal of the catalyst by filtration and, then, the extraction of the sufone compounds present in the organic phase with water. Interestingly, this process allowed the complete extraction of the DBT-suflone derivatives to the water phase leading to an organic phase with a sulfur content below the detection limit of our analytical system $\left(<4 \mathrm{mg} \mathrm{L}^{-1}\right.$ of $\left.\mathrm{S}, \sim 5.3 \mathrm{ppmw} \mathrm{S}\right)$. It is worthy to mention that the maximum sulfur content according to the legislation should be lower than 10 ppmw. ${ }^{30,31}$

\section{Experimental section}

Materials: DBT ( $\geq 99 \%$ ), DBT-sulfone (97\%), 4-methylDBT (96 $\%)$ and 4,6-dimethyIDBT (97 \%), tert-butylhydroperoxide (TBHP) solution in decane ( $5.5 \mathrm{M}), n$-decane, $n$-dodecane and $n$-tetradecane were of analytical grade and supplied by SigmaAldrich. The other reagents and solvents used in this work were analytical or HPLC grade.

Catalyst preparation: MIL-101(Cr) and MIL-101(Fe) were prepared and characterized as previously reported by our group ${ }^{23}$ following well-established procedures by Ferey ${ }^{9}$ and others. ${ }^{17,}{ }^{46}$ Briefly, a mixture of terephthalic acid $(0.25 \mathrm{~g}, 1.5$ $\mathrm{mmol}), \mathrm{Cr}\left(\mathrm{NO}_{3}\right)_{3} \cdot 9 \mathrm{H}_{2} \mathrm{O}(0.4 \mathrm{~g}, 1 \mathrm{mmol})$ and $\mathrm{HF}(10 \mu \mathrm{l})$ in water $(8 \mathrm{~mL})$ was placed in a Teflon-lined autoclave and heated at $200{ }^{\circ} \mathrm{C}$ for $8 \mathrm{~h}$. The resulting precipitate was purified by several washings with DMF and, then, with ethanol at $70{ }^{\circ} \mathrm{C}$ for $2 \mathrm{~h}$.

MIL-101(Fe) was prepared by dissolving terephthalic acid (0.206 g, $1.25 \mathrm{mmol}$ ) and $\mathrm{FeCl}_{3} \cdot 6 \mathrm{H}_{2} \mathrm{O}(0.675 \mathrm{~g}, 2.5 \mathrm{mmol})$ in DMF (15 mL) in a Teflon-lined autoclave and, then, heating the system at $135^{\circ} \mathrm{C}$ for $8 \mathrm{~h}$. The resulting precipitate was purified by double extraction with ethanol by stirring the powder in this alcohol (10 times in weight) at $60{ }^{\circ} \mathrm{C}$ for $2 \mathrm{~h}$.

Catalytic experiments: In a typical experiment a certain catalyst amount was added to a round-bottom flask $(25 \mathrm{~mL})$. When required, activation of MIL-101 catalyst was carried out by heating at $150{ }^{\circ} \mathrm{C}$ under vacuum for $6 \mathrm{~h}$. Subsequently, the reaction temperature was fixed $\left(110,120,130\right.$ or $\left.140{ }^{\circ} \mathrm{C}\right)$ and the required reaction atmosphere was obtained by purging the system with a balloon containing $\mathrm{O}_{2}$, air or argon under atmospheric pressure. The reaction time started by addition of a solution of DBT ( $200 \mathrm{mg} \mathrm{L}^{-1}$ of S) to the preheated roundbottom flask. As reaction solvents, $n$-dodecane as model of real hydrocarbons mixtures or commercial diesel (Repsol) were used.

Selective radical quenching experiments using TEMPO, $p$ benzoquinone or DMSO were carried out as described above but in the presence of $20 \mathrm{~mol} \%$ of these reagents respect to the initial DBT.

Liquid-liquid extraction. At the end of the reaction the catalyst was removed from the organic phase by filtration through a Nylon filter $(0.2 \mu \mathrm{m})$. Then, the organic phase $(10 \mathrm{~mL})$ was extracted with distilled water $(3 \times 10 \mathrm{~mL})$ using a separating funnel. This process resulted efficient for the complete DBTSufone extraction from the organic to the aqueous phase.

Product analysis: Aliquots of the reaction mixture at different times were diluted in a solution of anisole containing a known amount of nitrobenzene as external standard. Then, the samples were filtered to remove the catalyst and, then, analyzed by gas chromatography (GC) using a flame ionization detector. Quantification was carried by using calibration curves of commercial samples and nitrobenzene as standard.

To evaluate the metal leaching at the end of the reaction the solid catalyst was filtered through a $0.2 \mu \mathrm{m}$ nylon filter. Subsequently, an $\mathrm{HNO}_{3}$ aqueous solution ( $30 \mathrm{~mL}, 3 \mathrm{M}$ ) was put in contact with the organic phase reaction and the system magnetically stirred and heated at $80^{\circ} \mathrm{C}$ for $24 \mathrm{~h}$. The presence of $\mathrm{Cr}$ in the aqueous phase was finally analyzed using a ICP-OES instrument.

Raman measurements: Raman spectra were recorded with a $514 \mathrm{~nm}$ laser excitation on a Renishaw Raman Spectrometer ("Refelx") equipped with a CCD detector. The laser power on the sample was $25 \mathrm{~mW}$ and a total of 20 acquisitions were taken for each spectra. A commercial Linkam cell (THMS600) was used for collecting spectra under controlled conditions. Thus, the sample were first subjected to $\mathrm{N}_{2}$ flow $\left(20 \mathrm{ml} \mathrm{min}^{-1}\right.$ ) at $120{ }^{\circ} \mathrm{C}$ for $\sim 3 \mathrm{~h}$. Afterwards the gas was changed to $\mathrm{O}_{2}(20$ $\mathrm{ml} \mathrm{min}^{-1}$ ) at the same temperature. Finally, the temperature was lowered to $25{ }^{\circ} \mathrm{C}$ in $\mathrm{O}_{2}$ flow and changed to $\mathrm{N}_{2}$. Spectra were collected at $25^{\circ} \mathrm{C}$ in $\mathrm{N}_{2}$ flow.

EPR measurements: MIL-101(Cr) or MIL-101(Fe) (5 mg) were added to a round-bottom flask $(25 \mathrm{~mL})$ containing PBN $\left(1,150 \mathrm{mg} \mathrm{L}^{-1}\right)$ dissolved in $n$-dodecane $(10 \mathrm{~mL})$. After sonication (15 $\mathrm{min})$ the system was purged with a balloon containing $\mathrm{O}_{2}$ and the system heated at 120 o C for $5 \mathrm{~h}$. Then, an aliquot was filtered (Nylon filter, $0.2 \mu \mathrm{m}$ ) and the sample purged with $\mathrm{N}_{2}$ before recording the EPR. EPR spectra were recorded using a Bruker EMX instrument with the following parameters: frequency $9.803 \mathrm{GHz}$, sweep width $3489.9 \mathrm{G}$, time constant $40.95 \mathrm{~ms}$, modulation frequency $100 \mathrm{kHz}$, modulation width $1 \mathrm{G}$, microwave power $19.92 \mathrm{~mW}$.

\section{Conclusions}

In the present manuscript it has been shown that MIL-101 is an efficient, stable and reusable promoter for the aerobic oxidation of DBT and its methyl substituted derivatives in alkanes. The reaction exhibits and induction period that is related to the solvent diffusion and formation of the first reactive oxygen species, probably $\mathrm{O}_{2}{ }^{--}$or $\mathrm{HOO}$ that initiate a radical chain autooxidation mechanism. Evidence for the involvement of these species has been obtained by EPR spectroscopy after trapping the $\mathrm{PBN}-\mathrm{OOH}$ adduct and by Raman spectroscopy of $\mathrm{MIL}-101(\mathrm{Cr})$ exposed to $\mathrm{O}_{2}$. Considering that in the literature hydroperoxides have been proposed for the desulfuration of fuels, the present results can constitute an advantageous alternative based on the use of molecular oxygen as oxidant and a stable solid material as promoter.

\section{Acknowledgements}


Financial support by the Spanish Ministry of Economy and Competitiveness (Severo Ochoa and CTQ2012-32315) is gratefully acknowledged. Generalidad Valenciana is also thanked for funding (Prometeo 2012/013 and GV/2013/040)

\section{Notes and references}

1 1. H. Furukawa, Cordova, K.E., O'Keeffe, M., Yaghi, O.M., Science, 2013, 341, 1230444.

2 2. A. Corma, H. Garcia and F. X. Llabrés i Xamena, Chem. Rev., 2010, 110, 4606-4655.

3 3. Y.-Z. Yang, X.-G. Liu and B.-S. Xu, New Carbon Mater., 2014, 29, 1-14.

4 4. J. Gascon, A. Corma, F. Kapteijn and F. X. Llabrés i Xamena, ACS Catal., 2014, 4, 361-378.

5 5. M. Yoon, R. Srirambalaji and K. Kim, Chem. Rev., 2012, 112, 1196-1231.

6 6. A. Dhakshinamoorthy, M. Alvaro and H. Garcia, Catal. Sci. Technol., 2011, 1, 856-867.

7 7. J. Lee, O. K. Farha, J. Roberts, K. A. Scheidt, S. T. Nguyen and J. T. Hupp, Chem. Soc. Rev., 2009, 38, 1450-1459.

8 8. S. Kitagawa, R. Kitaura and S.-I. Noro, Angew. Chem. Int. Ed., 2004, 43, 2334-2237.

9 9. G. Ferey, C. Mellot-Draznieks, C. Serre, F. Millange, J. Dutour, S. Surble and I. Margiolaki, Science, 2005, 309, 2040-2042.

10 10. D.-Y. Hong, Y. K. Hwang, C. Serre, G. Férey and J.-S. Chang, Adv. Func. Mater., 2009, 19, 1537-1552.

11 11. A. Dhakshinamoorthy and H. Garcia, Chem. Soc. Rev., 2012, 41, 5262-5284.

12 12. T. Devic and C. Serre, Chem.Soc.Rev.,, 2014, 43, 60976115.

13 13. K. Brown, S. Zolezzi, P. Aguirre, D. Venegas-Yazigi, V. Paredes-García, R. Baggio, M. Novak and E. Spodine, Dalton Trans., 2009, 1422-1427.

14 14. A. Dhakshinamoorthy, M. Alvaro and H. Garcia, J. Catal., 2009, 267, 1-4.

15 15. L. Hamidipour and F. Farzaneh, Reac. Kinet. Mech. Cat., 2013, 109, 67-75.

16 16. O. A. Kholdeeva, I. Y. Skobelev, I. D. Ivanchikova, K. A. Kovalenko, V. P. Fedin and A. B. Sorokin, Catal. Today, 2014, 238, 54-61.

17 17. N. V. Maksimchuk, K. A. Kovalenko, V. P. Fedin and O. A. Kholdeeva, Chem. Comm., 2012, 48, 6812-6814.

18 18. C. Song, Catal. Today, 2003, 86, 211-263.

19 19. J. Sun, G. Yu, Q. Huo, Q. Kan and J. Guan, RSC Adv., 2014, 4, 38048-38054.

20 20. Z. Saedi, S. Tangestaninejad, M. Moghadam, V. Mirkhani and I. Mohammadpoor-Baltork, Catal. Comm., 2012, 17, 18-22.

2121 I. Y. Skobelev, K. A. Kovalenko, V. P. Fedin, A. B. Sorokin and O. A. Kholdeeva, Kinet. Catal., 2013, 54, 607-614.

2222 . I. Y. Skobelev, A. B. Sorokin, K. A. Kovalenko, V. P. Fedin and O. A. Kholdeeva, J. Catal., 2013, 298, 61-69.

23 23. A. Santiago-Portillo, S. Navalon, F. Cirujano, F. Llabrés Xamena, M. Alvaro and H. Garcia, ACS Catal. (DOI: 10.1021/acscatal.5b00411), 2015.
24 24. A. Dhakshinamoorthy, M. Alvaro, P. Concepcion and H. Garcia, Catal. Comm., 2011, 12, 1018-1021.

25 25. A. Dhakshinamoorthy, M. Alvaro and H. Garcia, J. Catal., 2012, 289, 259-265.

26 26. A. Dhakshinamoorthy, M. Alvaro and H. Garcia, ChemCatChem, 2010, 2, 1438-1443.

27 27. A. Dhakshinamoorthy, M. Alvaro and H. Garcia, ACS Catal., 2011, 1, 836-840.

28 28. A. Dhakshinamoorthy, M. Alvaro and H. Garcia, ACS Catal., 2011, 1, 48-53.

29 29. O. A. Kholdeeva, Catal. Sci. Technol., 2014, 4, 18691889.

30 30. V. C. Srivastava, RSC Adv., 2012, 2, 759-783.

31 31. B. Van de Voorde, B. Bueken, J. Denayer and D. De Vos, Chem. Soc. Rev., 2014, 43, 5766-5788.

32 32. N. D. McNamara, G. T. Neumann, E. T. Masko, J. A. Urban and J. C. Hicks, J. Catal., 2013, 305, 217-226.

33 33. D. Wang, E. W. Qian, H. Amano, K. Okata, A. Ishihara and T. Kabe, Appl. Catal. A: Ge, 2003, 253, 91-99.

3434 K.-B. Cho, H. Kang, J. Woo, J. Y. Park, M. S. Seo, J. Cho and W. Nam, Inorg. Chem., 2014, 53, 645-652.

35 35. D. P. Serrano, R. Sanz, P. Pizarro, I. Moreno and S. Medina, App. Catal. B-Environ., 2014, 146, 35-42.

36 36. Y. Liu, S. Liu, S. Liu, D. Liang, S. Li, Q. Tang, X. Wang, J. Miao, Z. Shi and Z. Zheng, ChemCatChem, 2013, 5, 3086-3091.

37 37. O. González-García and L. Cedeño-Caero, Catal. Today, 2012, 148, 42-48.

38 38. V. Chandra Srivastava, RSC Adv., 2012, 2, 759-783.

3939. M. Yu, N. Zhang, L. Fan, C. Zhang, X. He, M. Zheng and Z. Li, Rev. Chem. Eng., 2015, 31 27-43.

40 40. A. Samokhvalov, Tatarchuk, B.J., Catal. Rev. Sci. Eng., 2010, 52, 381-410.

41 41. S. Mikhail, T. Zaki and L. Khalil, Appl. Catal. A.-Gen., 2002, 227 265-278.

42 42. Y. C. Zhang, X. W. Yang, D. Li and P. Na, Adv. Mater. Res., 2014, 237-241.

43 43. Y. Zhang, A. Chen, C. Li, M. Luo and Z. Xu, Adsorpt. Sci. Technol., 2011, 29, 197-209.

44 44. S. G. McKinley and R. J. Angelici, Chem. Comm., 2003, 9.

45 45. K. A. Cychosz, A. G. Wong-Foy and A. J. Matzge, J. Am. Chem. Soc., 2008, 130, 6938-6939.

46 46. K. M. L. Taylor-Pashow, J. D. Della Rocca, Z. Xie, S. Tran and W. Lin, J. Am. Chem. Soc., 2009, 009, 14261-14263.

47 47. L. Helm and A. Merbach, E., Chem. Rev., 2005, 105, 1923-1959.

48 48. P. Horcajada, H. Chevreau, D. Heurtaux, F. Benyettou, F. Salles, T. Devic, A. Garcia-Marquez, C. Yu, H. Lavrard, C. L. Dutson, E. Magnier, G. Maurin, E. Elkaïmd and C. Serre, Chem. Commun., 2014, 50, 6872--6874.

49 49. A. Vimont, F. Thibault-Starzyk and M. Daturi, Chem. Soc. Rev., 2010, 39, 4928-4950.

50 50. P. Valvekens, F. Vermoortele and D. De Vos, Catal. Sci. Technol., 2013, 3, 1435-1445. 
51 51. H. V. Leclerc, A.; Lavalley, J. C.; Daturi, M.; Wiersum, A. D.; Llewellyn, P. L.; Horcajada, P.; Ferey, G.; Serre, C., Phys. Chem. Phys. C 2011, 13, 11748-11756.

52 52. A. Vimont, F. Thibault-Starzyk and M. Datur, Chem. Soc. Rev., 2010, 39, 4928-4950.

53 53. J. W. Yoon, Y.-K. Seo, Y. K. Hwang, J.-S. Chang, H. Leclerc, S. Wuttke, P. Bazin, A. Vimont, M. Daturi, E. Bloch, P. L. Llewellyn, C. Serre, P. Horcajada, J.-M. Grenche, A. E. Rodrigues and G. Férey, Angew. Chem. Int. Ed., 2010, 49, 5949-5952.

$5454 . \quad$ T. K. Trung, N. A. Ramsahye, P. Trens, N. Tanchoux, C. Serre, F. Fajula and G. Férey, Micropor. Mesopor. Mat., 2010, 134, 134-140.

55 55. J. Kim, S. Bhattacharjee, K.-E. Jeong, S.-Y. Jeong and W. S. Ahn, Chem. Commun., 2009, 3904-3906.

56 56. F. X. Llabrés i Xamena, O. Casanova, R. Galiasso Tailleur, H. Garcia and A. Corma, J. Catal., 2008, 255, 220-227.

57 57. A. Ansari, P. Jayapal and G. Rajaraman, Angew. Chem. Int. Ed., 2015, 54, 564 -568.

58 58. J. Cho, J. Woo and W. Nam, J. Am. Chem. Soc., 2010, 132, 5958-5959.

59 59. A. Dhakshinamoorthy, S. Navalon, M. Alvaro and H. Garcia, ChemSusChem, 2012, 5, 46-64.

60 60. M. J. Burkitt and R. P. Mason, Proc. Natl. Acad. Sci. USA, 1991, 88, 8440-8444.

61 61. R. Martin, S. Navalon, J. J. Delgado, J. J. Calvino, M. Alvaro and H. Garcia, Chem. Eur. J., 2011, 17, 94949502.

62 62. Z. Y. Diao, L. L. Han, Z. X. Wang and C. C. Dong, Phys. chem. B., 2005, 109, 5739-5745.

63 63. J. Guzman, S. Carrettin and A. Corma, J. Am. Chem. Soc., 2005, 127, 3286-3287.

64 64. R. Q. Long, Y. P. Huang and H. L. J. Wan, Raman Spectrosc., 1997, 28, 29-32.

65 65. S. Otsuki, T. Nonaka, N. Takashima, W. Qian, A. Ishihara, T. Imai and T. Kabe, Energ. Fuel., 2000, 14, 1232-1239.

66

67

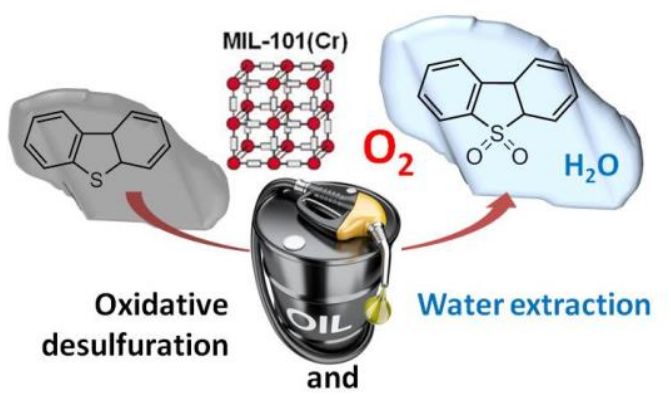

Table of contents. MIL-101(Cr) promotes the oxidative desulfuration of dibenzothiophenes by air. 